\title{
Elevated exosome-derived miRNAs predict osimertinib resistance in non-small cell lung cancer
}

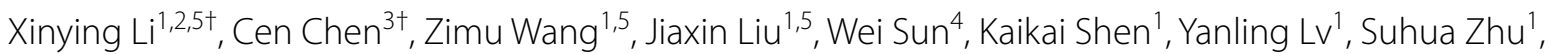
Ping Zhan ${ }^{1,5}$, Tangfeng L LV ${ }^{1,5^{*}}$ and Yong Song ${ }^{1,5^{*}}$ (D)

\begin{abstract}
Background: Non-small cell lung cancer (NSCLC) patients with epidermal growth factor receptor (EGFR) mutations will inevitably develop drug resistance after being treated with the third-generation EGFR-tyrosine kinase inhibitor (TKI), osimertinib. Recently, the drug resistance information transmitted by exosomal miRNAs has attracted much attention. However, the mechanism of exosome-derived miRNAs in osimertinib resistance remains unexplored.

Methods: We extracted and sequenced exosomes from the supernatant of the osimertinib-resistant cell line, H1975-OR, and the sensitive cell line, H1975. The results were compared with plasma exosome sequencing before and after the appearance of drug resistance in three NSCLC clinical patients treated with oral osimertinib. Exosomederived miRNAs that had significantly increased expression levels after osimertinib resistance were screened for expanded validation in other 64 NSCLC patients.
\end{abstract}

Results: Cluster analysis of the target genes revealed that exosomal miRNAs participate in osimertinib resistance mechanisms through the activation of bypass pathways (RAS-MAPK pathway abnormality and PI3K pathway activation). Exosome-derived miR-184 and miR-3913-5p expression levels increased significantly after the onset of osimertinib resistance. Exosomal miR-3913-5p was associated with TNM stage, platelet count, tumor marker carcinoembryonic antigen, and distant metastases. In patients with EGFR exon 21 L858R mutation, the increased expression levels of miR-184 and miR-3913-5p derived from serum exosomes indicated osimertinib resistance. Similarly, for T790M-positive patients, the level of exosome-derived miR-3913-5p can be used as a predictive marker for osimertinib resistance.

Conclusions: The expression levels of miR-184 and miR-3913-5p derived from exosomes in the peripheral blood of NSCLC patients could be used as biomarkers to indicate osimertinib resistance.

Keywords: Osimertinib (AZD9291), Exosome, miRNAs, Bypass pathway, NSCLC

*Correspondence: bairoushui@163.com; yong.song@nju.edu.cn ${ }^{\dagger}$ Xinying Li and Cen Chen contributed equally to this work 'Department of Respiratory and Critical Care Medicine, Jinling Hospital, Nanjing University School of Medicine, 305 East Zhongshan Road, Nanjing 210002, Jiangsu, China

Full list of author information is available at the end of the article

\section{Background}

Epidermal growth factor receptor (EGFR) mutations are the most common genetic mutations in non-small cell lung cancer (NSCLC) [1]. The PIONEER study (NCT01185314) [2] showed that the EGFR mutation rate in the Chinese population reached $50.2 \%$. Among them, exon 19 deletion (48.9\%) and exon 21 L858R point mutation (45.4\%) were the most common [2, 3]. Usually, $50-60 \%$ of the NSCLC patients with EGFR mutations original author(s) and the source, provide a link to the Creative Commons licence, and indicate if changes were made. The images or other third party material in this article are included in the article's Creative Commons licence, unless indicated otherwise in a credit line to the material. If material is not included in the article's Creative Commons licence and your intended use is not permitted by statutory regulation or exceeds the permitted use, you will need to obtain permission directly from the copyright holder. To view a copy of this licence, visit http://creativecommons.org/licenses/by/4.0/. The Creative Commons Public Domain Dedication waiver (http://creativeco mmons.org/publicdomain/zero/1.0/) applies to the data made available in this article, unless otherwise stated in a credit line to the data. 
develop acquired resistance to tyrosine kinase inhibitors (TKI) after first- and second-generation EGFR-TKI therapy [4], especially those with p.Thr790Met (T790M) point mutation in exon 20. The FLAURA study, which received wide attention at the 2019 European Society for Medical Oncology (ESMO) congress, confirmed that EGFR-mutant NSCLC patients treated with the thirdgeneration EGFR-TKI osimertinib [5] had a 6.8 months prolongation of overall survival (OS) compared to patients receiving gefitinib or erlotinib therapy [6]. However, patients treated with osimertinib inevitably develop resistance.

Exosomes [7] are extracellular vesicles measuring $30-150 \mathrm{~nm}$ that transmit biological information and participate in cell-mediated biological activities by releasing large amounts of proteins, RNA, lipids, and other biomolecules to the extracellular environment [8]. MicroRNAs (miRNAs) [9] in exosomes are not digested by RNases, allowing them to be transported between cells and participate in epigenetics [10]. Existing literatures have shown that exosomal miRNAs (exo-miRNAs) secreted by tumor cells play an important role in drug resistance [11, 12]. Whether exosomes are involved in resistance to the third-generation EGFR-TKI osimertinib remains unclear.

In this study, we investigated whether miRNAs in exosomes, especially before and after resistance to osimertinib, transmit relevant information between tumor cells, leading to changes in the sensitivity of patients. After collecting exosomes from the supernatant of osimertinib drug-resistant and drug-sensitive cells and comparing plasma exosomes of matched patients, a nextgeneration RNA-seq test was performed to identify key miRNAs. Later, we verified the role of key miRNAs in osimertinib resistance in larger population samples in an attempt to elucidate the mechanism of exo-miRNAs involved in osimertinib resistance. We hope to provide directions for the treatment of NSCLC patients with osimertinib resistance.

\section{Materials and methods \\ Cell culture and establishment of resistant strains}

Lung adenocarcinoma cell line H1975 cells (EGFR, L858R and T790M) were purchased from the Institute of Biochemistry and Cell Biology, Chinese Academy of Sciences (Shanghai, China). Osimertinib was obtained from Selleck Chemicals (Houston, TX, USA). The osimertinib-resistant cell line, H1975-OR, was induced and cultivated as previously reported [13]. All cells were cultured in RPMI 1640 medium containing 10\% fetal bovine serum (FBS) and antibiotics (100 units/mL penicillin and $100 \mu \mathrm{g} / \mathrm{mL}$ streptomycin). All cells were incubated at $37^{\circ} \mathrm{C}$ in $5 \% \mathrm{CO}_{2}$ humidified air.
Cell proliferation was measured in three replicates using the MTT method as described previously [14]. A non-linear regression function was used to fit the doseresponse curve in GraphPad Prism 5.0. After the $\mathrm{IC}_{50}$ values of the semi-inhibition rates of H1975 and H1975-OR cells were obtained, the drug resistance index was calculated. Both MTT and dimethyl sulfoxide (DMSO) were purchased from Sigma-Aldrich (St. Louis, MO, USA). RPMI 1640 medium, fetal bovine serum (FBS), penicillin, and streptomycin were purchased from Gibco Life Technologies (Grand Island, NY, USA).

\section{Patient selection and sample collection}

A total of 67 NSCLC patients were included in this study and were observed in the Department of Respiratory and Critical Care Medicine at Jinling Hospital from December 2018 to October 2019. Three patients had a pair of blood samples collected each, before and after the appearance of resistance to osimertinib, to be compared with the H1975 cells samples. The samples of the remaining 64 patients were used for extended screening after identifying target miRNAs. Among these patients, 37 were resistant to gefitinib but had not yet developed osimertinib resistance, and 27 had Osimertinib resistance. In addition, 10 healthy people were recruited from the physical examination center of Jinling Hospital. All participants provided written informed consent. This study was approved by the ethics committee of Jinling Hospital (Ethical code: 2017NZGKJ-030).

\section{Isolation of exosomes}

After reaching $80 \%$ confluency in a Petri dish, H1975 and H1975-OR cells were cultured in complete RPMI1640 medium without added serum. The cell culture supernatant was collected $24 \mathrm{~h}$ later, and the exosomes were separated using differential ultracentrifugation as described previously [15]. To remove cell debris, the supernatant was centrifuged at $300 \times g$ for $5 \mathrm{~min}$, at $3000 \times g$ for $10 \mathrm{~min}$, and, lastly, at $10,000 \times g$ for $30 \mathrm{~min}$. The supernatant was then passed through a $0.22-\mu \mathrm{m}$ filter (Millipore, Burlington, MA, USA). The filtered supernatant was transferred to a clean ultracentrifuge tube and ultracentrifuged at $4{ }^{\circ} \mathrm{C}, 120,000 \times g$ for $70 \mathrm{~min}$. After discarding the supernatant, the pellet was resuspended in an appropriate amount of sterile phosphate-buffered saline $(1 \times \mathrm{PBS})$. The extracted exosomes were used for immediate downstream experiments or stored in a $-80{ }^{\circ} \mathrm{C}$ refrigerator.

Serum samples were extracted using a Total Exosome Isolation Kit (from serum) $(4,478,360$, Invitrogen, Carlsbad, CA, USA,). The required volume of clear serum was transferred to a new test tube and 0.2 volume of total exosome isolation (from serum) reagent was added. The 
serum and reagent were mixed, through eddy current or an up-and-down pipe motion, to make the solution turbid. After incubation at $4{ }^{\circ} \mathrm{C}$ for $30 \mathrm{~min}$, the samples were centrifuged at $10,000 \times g$ for $10 \mathrm{~min}$. The supernatant was aspirated and discarded. The exosomes, which were contained in the granules at the bottom of the test tube, were resuspended in half the volume of the initial serum volume of sterile PBS $(1 \times)$.

\section{Transmission electron microscopy (TEM), size distribution analysis, and western blot}

Exosome morphology was observed using TEM. The exosome suspension was mixed with an equal volume of $4 \%$ paraformaldehyde, and $10 \mu \mathrm{L}$ of the mixture was placed on a clean copper grid (RT) at room temperature. Uranyl acetate staining was negative. The images were acquired by observation with a JEOL 1200EX TEMSCAN microscope. The exosomal suspensions were analyzed for particle size using dynamic light scattering (DLS) (Nanosizer ${ }^{\mathrm{TM}}$ instrument, Malvern Instruments, Malvern, UK).

The extracted exosomes were resuspended in cell lysate (Beyotime, Nantong, China) supplemented with 1\% PMSF. The protein concentration of exosomes was determined using a Pierce BCA protein detection kit (Thermo Fisher Scientific, Rockford, IL, USA). Sodium dodecyl sulfate polyacrylamide gel electrophoresis (SDS-PAGE) (12\%) were prepared with $20 \mu \mathrm{g}$ of protein on each sample. Anti-CD63, anti-TSG101, anti- $\beta$-actin, and antiGAPDH were purchased from Abcam (Cambridge, UK). All antibodies used in western blot are diluted 1:1000.

\section{RNA-sequencing and raw data}

The total RNA in the exosomes was extracted using TRIzol reagent (Invitrogen, Carlsbad, CA, USA) according to the manufacturer's instructions. After performing the RNA extraction and quality control, the library was constructed and sequenced according to the literature. The raw data from small RNA-seq include linker sequences and sequencing low-quality sequences. In order to ensure the accuracy of the information analysis, the raw sequencing data were filtered to obtain clean data, and subsequent bioinformatics analysis was performed based on the clean data. The Qphred score (Qphred $=10 \log 10$ (e)) was used to represent the base quality value (Quality Score) to measure the quality of each base in the sequencing reads. The miRDeep2 software was used to analyze miRNA expression abundance. Cluster analysis and correlation analysis between samples (Pearson's correlation coefficient) were performed on the miRNA family in each sample. All raw sequencing data were uploaded to the SRI database. [Submission ID: SUB7187450; BioProject ID: PRJNA615931] (http://www.ncbi.nlm.nih.gov/biopr oject/615931).

\section{Target gene analysis}

The analysis was performed using DESeq2 (no biological duplicate samples use DESeq or edgeR); miRNAs with [logFoldChage] $>1$ and $p$ value $<0.05$ were selected as miRNAs with significant differences. A volcano map of the results of miRNA differential analysis and the clustered heat map of miRNA expression in the samples were drawn. TargetScan was used to calculate a weighted context ++ score to predict the target genes of different known miRNAs. These target gene functions were classified through a database established using the Gene Ontology (GO) Consortium, and a GO enrichment analysis was performed. At the same time, the first 10 target genes of each sample were selected for Kyoto Encyclopedia of Genes and Genomes (KEGG) pathway enrichment analysis according to the parameters described in the literature to identify the downstream molecular metabolic pathways of these target genes.

\section{Quantitative reverse transcription PCR}

The RNA extracted from the sera of patients was quantified and evaluated using NanoDrop ${ }^{\circledR}$ ND-2000 (Thermo Fisher Scientific, USA). We used the miRNA first-strand cDNA synthesis (tailing method) kit from Shanghai Sangon Biotech, using the method of Poly (A) tailing reaction and cDNA synthesis reaction simultaneously. After following the manufacturer's instructions, all cDNA products of the miRNAs were obtained and real-time PCR experiments were performed. In the RT-PCR, the universal downstream primer Universal PCR Primer R and the endogenous reference U6 (Universal U6 Primer F) in the above kit were used uniformly. The upstream primers of several miRNAs are as follows: miR-184: 5'-catGGACGG AGAACtGAtAAGGGt-3 '; miR-3913-5p: 5'-acggTTT GGGACTGATCTTGATGTCT-3'; miR-4746-5p: 5'-CCG GTCCCAGGAGAACC-3'; miR-3614-5p: 5'-CCACTT GGATCTGAAGGCTGC-3'. RT-PCR was performed using an ABI 7500 real-time PCR system (Applied Biosystems, Foster City, CA, USA). The expression levels of all miRNAs were determined using the 2- $\Delta \Delta C$ T method: $\Delta \mathrm{CT}$ (target) $=\mathrm{CT}$ (target) $-\mathrm{CT}$ (U6) [16]. All the above experiments were performed twice.

\section{Statistical analysis}

The above data were mostly analyzed and mapped using the SPSS 22.0 system (SPSS, Inc. Chicago, IL, USA) and GraphPad Prism 5. The differences in the miRNA expression levels between the two groups were mainly determined using the Mann-Whitney rank-sum test for non-parametric data. The correlation between ExomiR-184 and Exo-miR-3913-5p with clinicopathological 
features was determined using the $\chi^{2}$ test. ROC curves were also used to determine the diagnostic value. $\mathrm{P}<0.05$ values indicate a statistical difference.

\section{Results}

Isolation of exosomes from cell supernatant and serum In the commonly used lung cancer cell lines, H1975 carries both EGFR-L858R drug-sensitive mutation and EGFR-T790M drug-resistant mutation. The drug-resistant strain H1975-OR was established by increasing the concentration of osimertinib. Six months later, the MTT method was used to determine the cell viability of the sensitive strain H1975 and the resistant strain H1975-OR (Fig. 1A). The average $\mathrm{IC}_{50}$ values of these two cells were $4636 \mathrm{nM}$ and 12,101 $\mathrm{nM}$, respectively $(\mathrm{p}=0.0215)$ (Fig. 1B); RI was 2.61. Circular vesicle-like exosomes were obtained from different cell supernatants after ultracentrifugation. Blood samples were collected from patients with EGFR mutations before and after osimertinib resistance. The exosomes were extracted using the kit. Exosomes were also identified using TEM (Fig. 1C), western blotting (Fig. 1D), and DLS analysis (Additional file 1: Fig. S1A, B).

\section{Functional enrichment analysis of differentially expressed miRNA target genes in exosomes}

The volcano map visually revealed the difference in miRNA expression of exosomes in the supernatant of

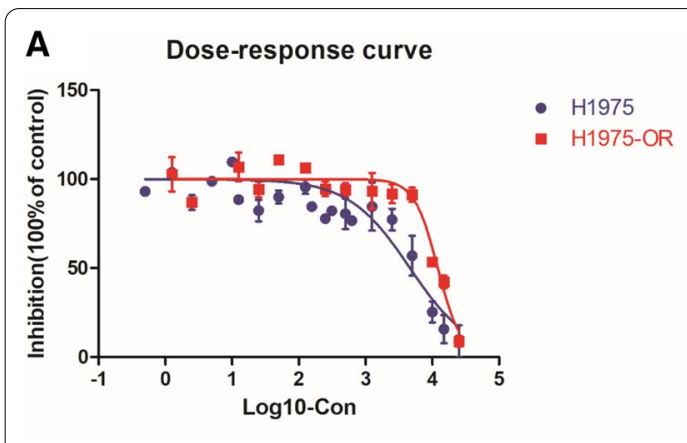

C

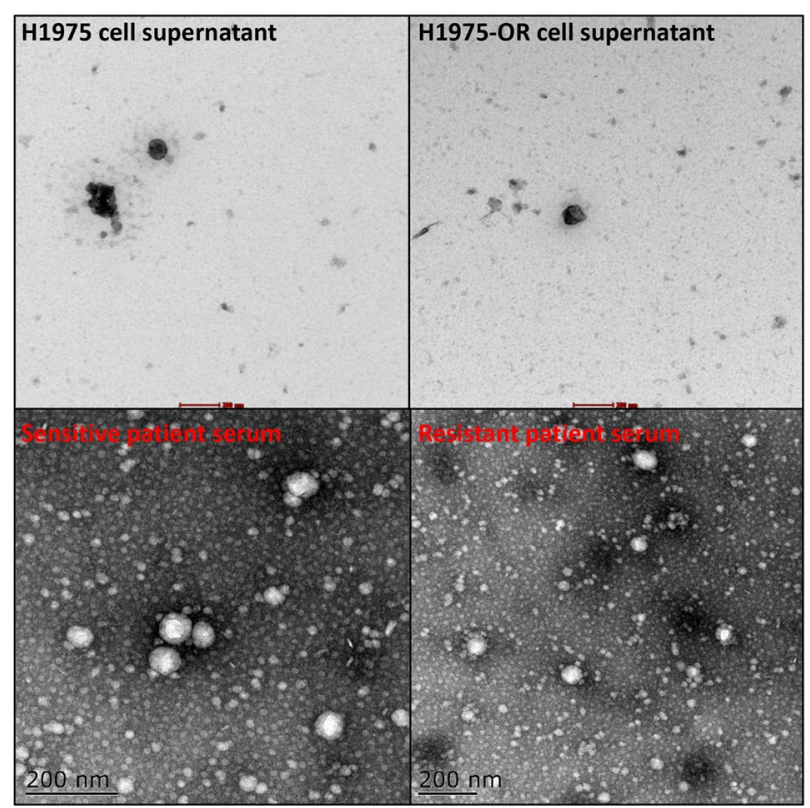

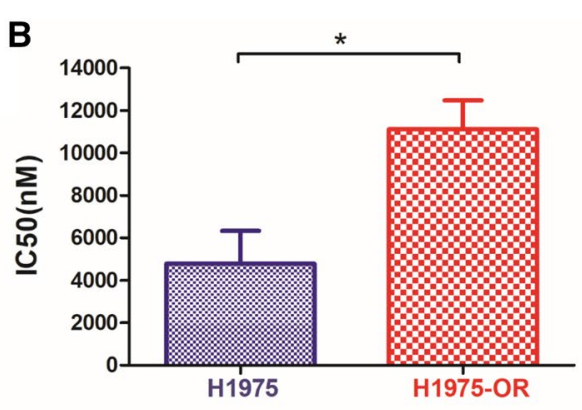

D

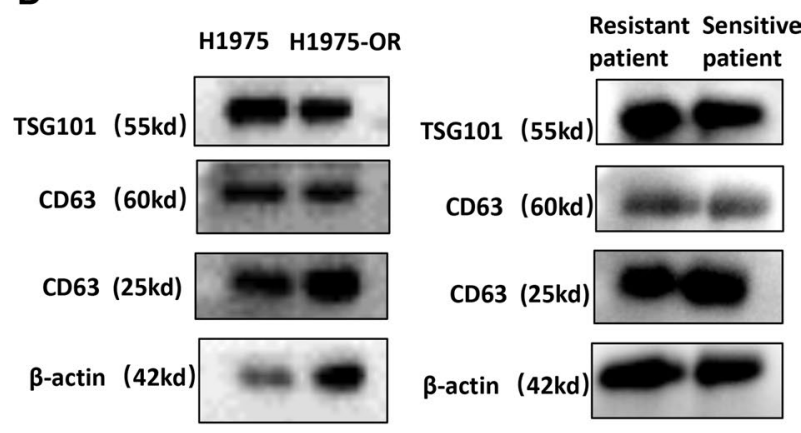

Fig. 1 Isolation and identification of exosomes. A The drug-resistant strain, H1975-OR, was obtained by continuous exposure of H1975 to osimertinib for 6 months. After incubating H1975-OR and H1975 cells with different concentrations of osimertinib for 72 h, the cell viability was measured using the MTT method. B The average $\mathrm{IC}_{50}$ values in $\mathrm{H} 1975$ and H1975-OR were $4636 \mathrm{nM}$ and 12,101 nM, respectively, and the difference was statistically significant ( $p=0.0215)$. C The exosomes (30-150 nm) extracted from the H1975, H1975-OR cell supernatant, the osimertinib-sensitive and osimertinib-resistant patients serum were observed using electron microscopy. Scale = $200 \mathrm{~nm}$. D Exosomes were validated by protein analysis in western blot 
A

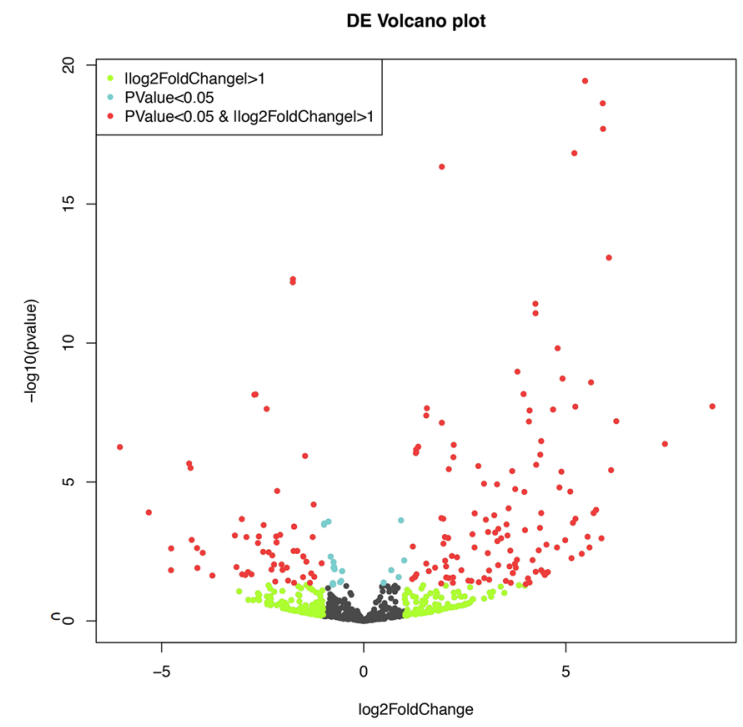

C

KEGG Pathway Enrichment

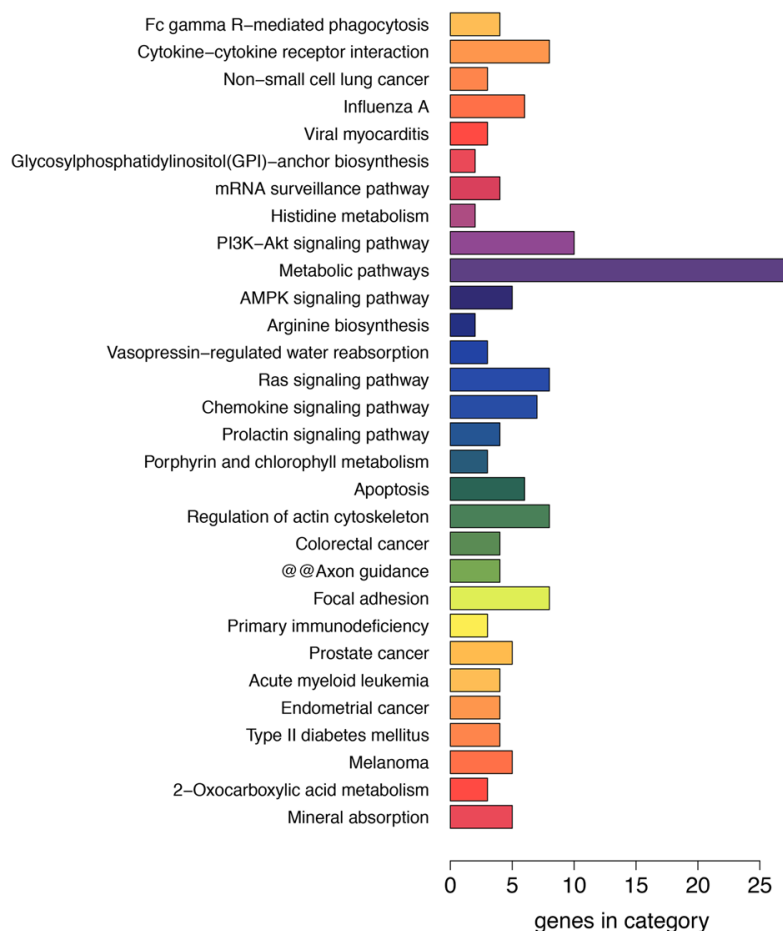

B

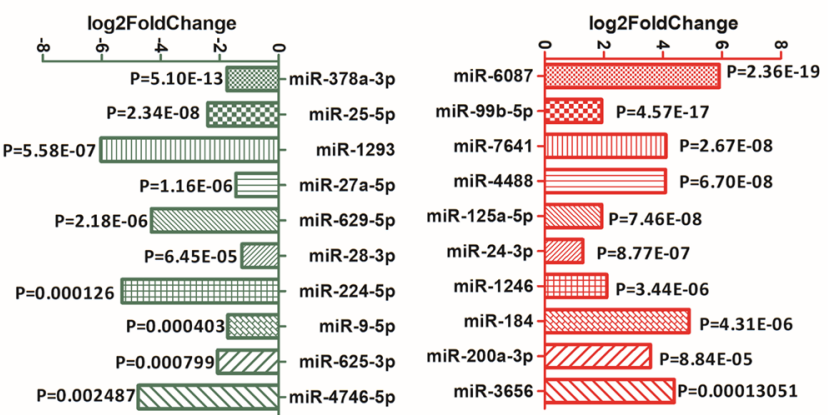

D

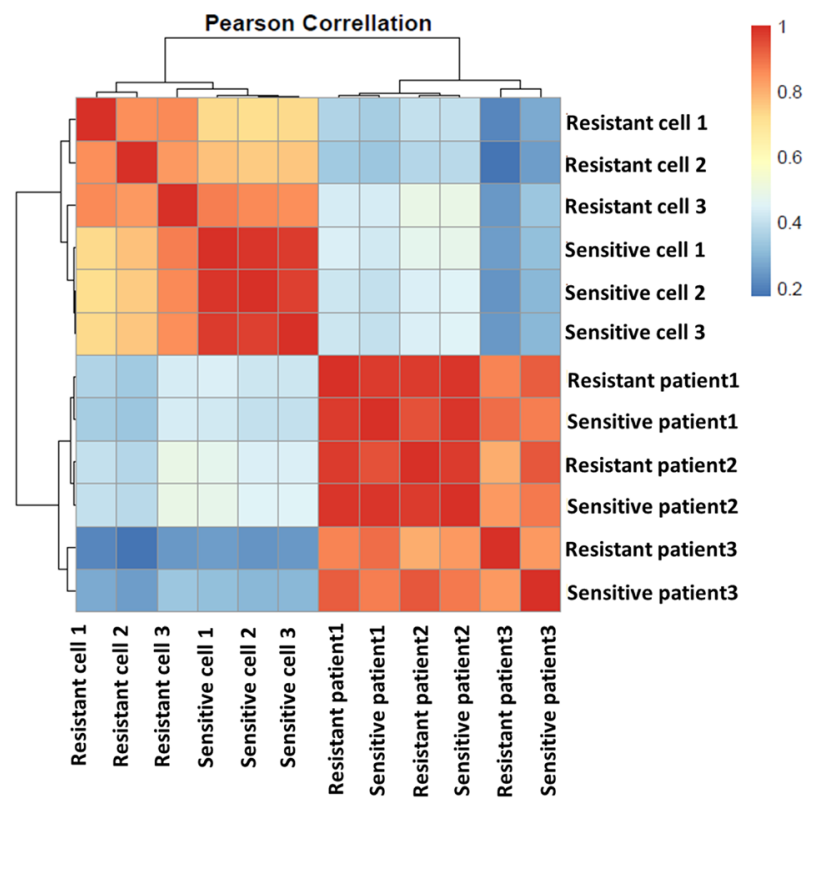

Fig. 2 Cluster analysis of miRNAs and target genes. A Volcano map of differentially expressed miRNAs. Each point in the volcano map represents a gene, and the red dots are significantly different genes that satisfy both $[\log F C]>1$ and FDR $<0.05$ thresholds. The blue dots are genes that only meet $\mathrm{FDR}<0.05$, and the green dots are genes that only meet [logFC] $>1$. B H1975-OR top 10 up-regulated miRNAs (red) and top 10 down-regulated miRNAs (green) compared with H1975. C KEGG pathway enrichment analysis of target genes for differentially expressed miRNA (histogram). The ordinate represents the name of the pathway, and the abscissa is the number of genes involved in each pathway. D Calculate the Pearson coefficient of cell supernatant and patient serum.. The correlation coefficient uses Pearson's correlation test (column, row), and the color of each square corresponds to the correlation coefficient value 
H1975 and H1975-OR cells (Fig. 2A). Among them, the expression levels of exosome miR-6087, miR-99b-5p, and miR-7641 in the H1975-OR supernatant were significantly higher than those in the H1975 supernatant. Other exo-miRNAs, such as miR-378a-3p, miR-25-5p, and miR1293, were significantly reduced (Fig. 2B). KEGG pathway enrichment was carried out for the top ten target genes of exosome miRNAs with differences between the two groups. The "metabolic pathway" involved the largest number of target genes, followed by the "PI3K-Akt signaling pathway," "Ras signaling pathway," "cytokinecytokine receptor interaction," "non-small cell lung cancer," and other pathways (Fig. 2C). The pathway analysis of novel differentially expressed miRNA target genes focused on the MAPK signaling pathway (Additional file 1: Figure S1). These pathways are also members of the bypass pathways involved in drug resistance in EGFRTKI therapy for NSCLC.

A 62-year-old male with NSCLC (Patient 2, Treat3 \& Control3) (left lung adenocarcinoma, EGFR exon 19 deletion) was treated with gefitinib. One year later, he started oral AZD9291 due to new brain and bone metastasis. The cancer progressed after 19 months. Blood samples were collected from the patient at the start of osimertinib treatment and after presenting resistance. Similarly, blood samples were collected from a 53-year-old female patient (left lung adenocarcinoma stage IVB, EGFR exon 21 L858R mutation) (Patient 1, Treat2 \& Control2) and a 58-year-old female patient (left lung adenocarcinoma stage IVB, EGFR exon 19 deletion) (Patient 3, Treat4 $\mathcal{E}$ Control4) before and after the appearance of resistance to osimertinib. Pearson's correlation coefficient (R2) was used to assess the correlation between exo-miRNAs from the cell supernatant and serum of patients, and the correlation heat map matrix was drawn (Fig. 2D).

\section{Elevated miR-184 and miR-3913-5p in exosomes after osimertinib resistance}

We performed an overlapping analysis of the differential miRNAs obtained by sequencing exosomes in the cultured cells supernatant (treat1) and the blood samples of three patients (treat2-4) using a Wayne map (Fig. 3A, B). Among the exo-miRNAs upregulated in the osimertinib-resistant group compared to those in the sensitive group, no common differential miRNAs were found to overlap in the four groups (Fig. 3A). However, in a pairwise comparison, it was found that miR-184 overlapped in treat 1 and treat4, miR-3913-5p appeared both in treat 3 and treat 4 , and miR-3656 co-occurred in treat 1 and treat2 (Fig. 3A). These three miRNAs were further studied. Similarly, miR-3614-5p, miR-4746-5p, and miR-378i were chosen as the target miRNAs in the down-regulated miRNA group (Fig. 3B).
In order to verify several significantly up-regulated exo-miRNAs in the RNA-seq results, we decided to expand the sample size for experiments. Serum samples from 37 NSCLC patients with gefitinib resistance and 27 patients with osimertinib resistance were collected as controls (GR) and experimental groups (OR). Using QPCR, we verified that the exosome miR-184 in the serum of drug-resistant patients was significantly high $(p=0.0325)$ (Fig. 3C), and exosome miR-3913-5p was significantly increased in the osimertinib-resistant group $(\mathrm{p}=0.0169)$ (Fig. 3D), which was consistent with the previous sequencing results.

\section{Increased miRNAs suggested that NSCLC patients were resistant to osimertinib}

Combining these two exo-miRNAs, it was found that these two miRNAs in the serum exosomes of NSCLC patients were significantly upregulated after osimertinib resistance $(p=0.0092)$ (Fig. 3E, F). The relationship between exo-miRNA expression levels and the clinicopathological characteristics of the patients (e.g., age, sex, smoking history, TNM stage, primary tumor size, platelet count (PLT), lactate dehydrogenase (LDH), carcinoembryonic antigen (CEA), and distant metastasis) is summarized in Tables 1 and 2. Exosome miR-184 was correlated with LDH levels $(\mathrm{p}=0.018)$ (Table 1$)$; exosome miR-3913-5p was associated with TNM stage $(\mathrm{p}=0.045)$, PLT $(p=0.024)$, tumor marker CEA $(p=0.045)$, distant metastases $(p=0.049)$, and, especially, bone metastasis $(p=0.03)$ (Table 2). However, there was no significant correlation between miRNA expression levels of the two exosomes and age, sex, smoking status, ECOG score, and primary tumor size.

\section{Exosomal-derived miRNAs predict osimertinib resistance in patients with EGFR exon21 L858R mutation.}

Of the 64 NSCLC patients, 28 possessed EGFR exon 19 deletions and 36 had mutations in the EGFR exon 21. In all patients with exon 19 deletion, there was no significant difference in the expression levels of serum exosomal miR-184 $(\mathrm{p}=0.776)$ and miR-3913-5p $(\mathrm{p}=0.631)$ between the groups before and after drug resistance (Additional file 1: Fig. S4A, B). However, in all patients with mutations in L858R in the EGFR exon 21, the serum expression level of exo-miR-184 was significantly elevated in osimertinib-resistant patients $(\mathrm{p}=0.0104)$ (Fig. 4A). Exo-miR-3913-5p $(\mathrm{p}=0.0085)$ was also significantly altered in patients with osimertinib resistance (Fig. 4B). ROC curve analysis showed that the AUC of exo-miR-184 was 0.736 , while the AUC of exo-miR3913-5p was 0.759 (Additional file 1: Fig. S4C, D), indicating that these two miRNAs play important roles in osimertinib resistance and were mainly involved in drug 


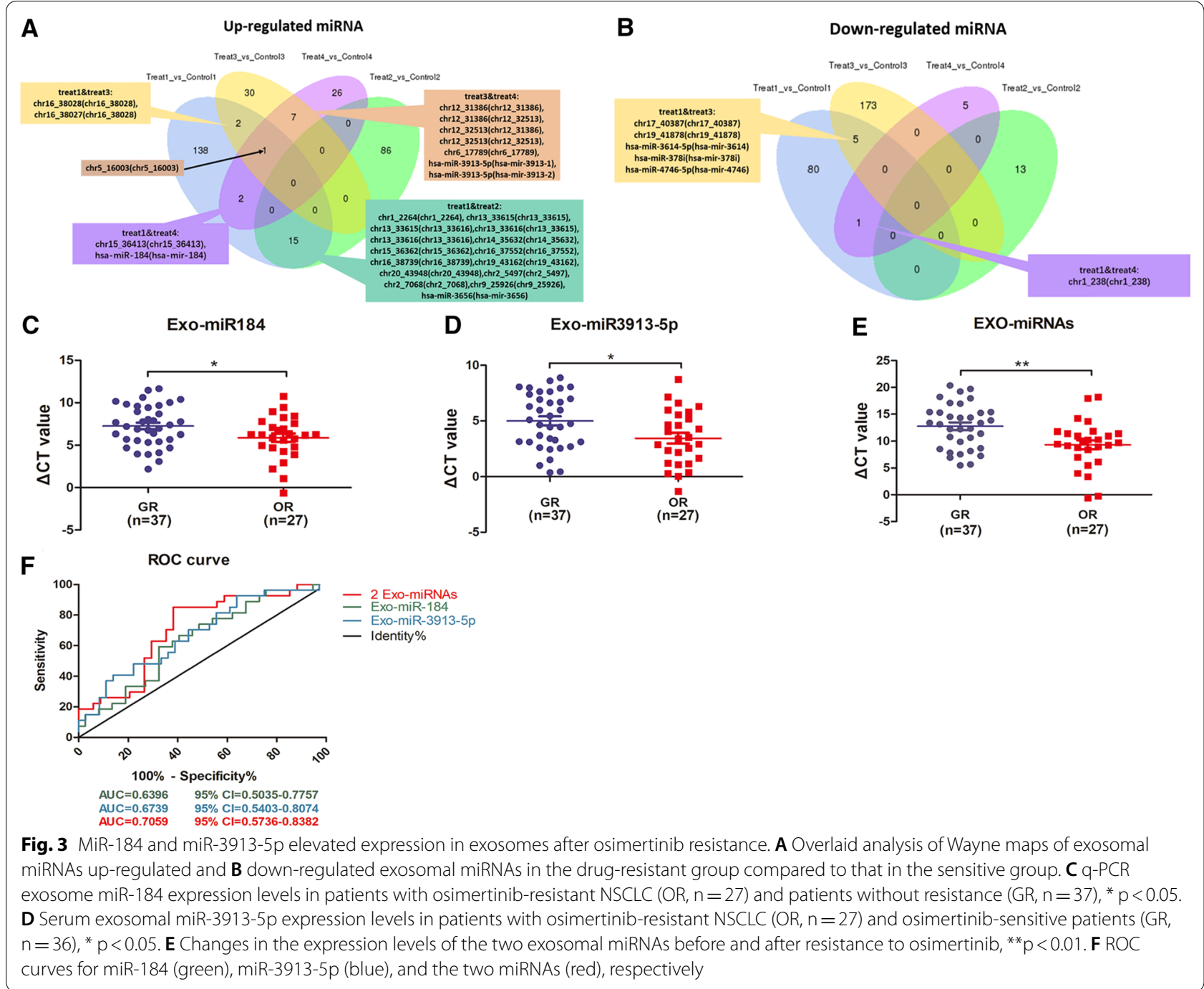

resistance in patients with EGFR exon 21 L858R. Because the sensitive group (GR) was selected for gefitinib-resistant patients, the criteria for clinical resistance in these patients were enlarged lesions or new organ metastases. After re-sequencing, most patients $(n=44)$ showed T790M-positive mutations in EGFR-TKI resistance, but a few patients $(n=20)$ were not T790M-positive (Additional file 1: Fig. S4). In T790M + osimertinib-resistant patients, the expression level of exo-miR-3913-5p was significantly increased ( $p=0.013)$ (Fig. $4 \mathrm{D})$, and the exomiR-184 expression level in the resistant group was also higher than that in the sensitive group, but the difference was not statistically significant ( $\mathrm{p}=0.065)$ (Fig. 4C).

\section{Discussion}

Osimertinib (AZD9291) is the first third-generation EGFR-TKI to be approved by the FDA and EMA for the treatment of NSCLC [5]. It is selective for EGFR-TKI sensitization and $7790 \mathrm{M}$ resistance mutations and has less effect on WT-EGFR [17]. However, similar to the findings observed after the use of other EGFR-TKIs, patients still inevitably develop resistance after receiving osimertinib, regardless of its first- or second-line use, which greatly limits the long-term clinical benefits of this targeted drug [18]. The mechanism of osimertinib resistance depends on the high tumor heterogeneity of NSCLC, which is divided into EGFR-dependent and EGFR-independent aspects [19]. Moreover, previous studies have shown that the resistance mechanism after first- or second-line use of osimertinib varies with clonal evolution [20]. Our study focused on patients with advanced NSCLC, who were treated with nonfirst-line osimertinib. Patients enrolled in the sensitive group (GR) were those who were sensitive to osimertinib after gefitinib resistance, while patients in the resistant group (OR) were those who had developed 
Table 1 Correlation between serum exosomal miR184 expression levels in patients with NSCLC and their clinicopathological characteristics. ( $\left.{ }^{*} p<0.05\right)$

\begin{tabular}{|c|c|c|c|c|}
\hline \multirow[t]{2}{*}{ Clinicopathological parameters } & \multirow{2}{*}{$\begin{array}{l}\text { Number of } \\
\text { patients(\%) }\end{array}$} & \multicolumn{3}{|c|}{ Exosomal miR-184 } \\
\hline & & Low & High & $P$ \\
\hline Age(years), Median & & & & 0.777 \\
\hline$<60$ & 33 & 15 & 18 & \\
\hline$\geq 60$ & 31 & 13 & 18 & \\
\hline Gender & & & & 0.899 \\
\hline Male & 28 & 12 & 16 & \\
\hline Female & 36 & 16 & 20 & \\
\hline Smoking history & & & & 0.353 \\
\hline Never smoking & 49 & 23 & 26 & \\
\hline Now/once smoking & 15 & 5 & 10 & \\
\hline ECOG Score & & & & 0.226 \\
\hline $0-1$ & 53 & 25 & 28 & \\
\hline$\geq 2$ & 11 & 3 & 8 & \\
\hline Primary tumor & & & & 0.705 \\
\hline$\leq 4 \mathrm{~cm}$ & 19 & 9 & 10 & \\
\hline$>4 \mathrm{~cm}$ & 45 & 19 & 26 & \\
\hline TNM & & & & 0.317 \\
\hline I-IVA & 25 & 9 & 16 & \\
\hline IVB & 39 & 19 & 20 & \\
\hline PLT & & & & 0.411 \\
\hline $\operatorname{Low}(\leq 146)$ & 13 & 7 & 6 & \\
\hline $\operatorname{High}(>146)$ & 51 & 21 & 30 & \\
\hline LDH & & & & $0.018^{*}$ \\
\hline $\operatorname{Low}(\leq 199)$ & 29 & 8 & 21 & \\
\hline $\operatorname{High}(>199)$ & 35 & 20 & 15 & \\
\hline CEA & & & & 0.129 \\
\hline $\operatorname{Low}(\leq 8.605)$ & 25 & 8 & 17 & \\
\hline $\operatorname{High}(>8.605)$ & 39 & 20 & 19 & \\
\hline Distant metastasis & & & & 0.164 \\
\hline No & 17 & 5 & 12 & \\
\hline Yes & 47 & 23 & 24 & \\
\hline Contralateral lung metastasis & & & & 0.069 \\
\hline No & 24 & 7 & 17 & \\
\hline Yes & 40 & 21 & 19 & \\
\hline Pleura metastasis & & & & 0.825 \\
\hline No & 33 & 14 & 19 & \\
\hline Yes & 31 & 14 & 17 & \\
\hline \multicolumn{5}{|l|}{ Bone metastasis } \\
\hline No & 23 & 9 & 14 & \\
\hline Yes & 41 & 19 & 22 & \\
\hline \multicolumn{5}{|l|}{ Brain metastasis } \\
\hline No & 36 & 14 & 22 & \\
\hline Yes & 28 & 14 & 14 & \\
\hline
\end{tabular}

clinical resistance to osimertinib. This ensured the homogeneity of these two sample groups.

The most common EGFR-dependent drug-resistance mechanism is C797S mutation occurring on exon 20 , which has previously been reported to account for $10-26 \%$ of all cases using osimertinib as secondline therapy [21]. In addition to C797S-adjacent G796 mutation, there are multiple mutation sites possibly involved, which include L792, L718, G719, G724, and EGF overexpression [22]. However, in our study, of the 27 patients with osimertinib resistance, only 3 (11.11\%) had a C797S mutation that was confirmed based on molecular testing. Of course, there were also several patients who did not undergo genetic testing again for financial reasons.

The most important EGFR-independent mechanisms of drug resistance are activation of bypass signals, abnormalities in downstream pathways, and histological transformation. The most common activated bypass pathways are MET amplification [23] and HER2 amplification [24]. The abnormality of the RAS-MAPK pathway is also an important mechanism of osimertinib resistance. OrtizCuaran et al. [25] confirmed that when acquired resistance to second-line osimertinib was developed, re-biopsy of the tumor revealed a KRAS G12S mutation. Kim et al. [26] reported a case of MAPK1 mRNA overexpression in a patient who received second-line treatment with osimertinib in advanced stages. This is consistent with our exosome sequencing results. As shown in Fig. 2C, the most obvious pathway enriched by the known differential exo-miRNA target gene in the supernatants of drug-resistant (H1975-OR) and sensitive (H1975) strains was the Ras signaling pathway. As shown in Additional file 1: Figure S2, the MAPK signaling pathway is the most abundant among the novel differential miRNA target genes with the largest number of genes involved. The above results indicated that the participation of exomiRNAs in osimertinib resistance was also related to abnormalities in the RAS-MAPK pathway. In addition, the PI3K pathway is involved in bypass activation. It is currently believed that PIK3CA mutation or amplification and PTEN deletion can lead to activation of the PI3K pathway [26]. In our study, the PI3K-Akt signaling pathway was observed in KEGG enrichment analysis. ExomiRNAs may convey osimertinib resistance information to affect the activation of the PI3K pathway. Osimertinib resistance is also related to changes in cell cycle genes, including cyclin D1, cyclin D2, cyclin E1, cyclin-dependent kinase (CDK) 4, and CDK6 [27]. In the sequencing results, exosome miR-6087 was significantly increased in the drug-resistant group. Its target gene was CCND1 (Additional file 1: Table S1), which encodes the cyclin D1 protein. This again demonstrated that exosomes 
Li et al. Cancer Cell Int $\quad$ (2021) 21:428

Page 9 of 14

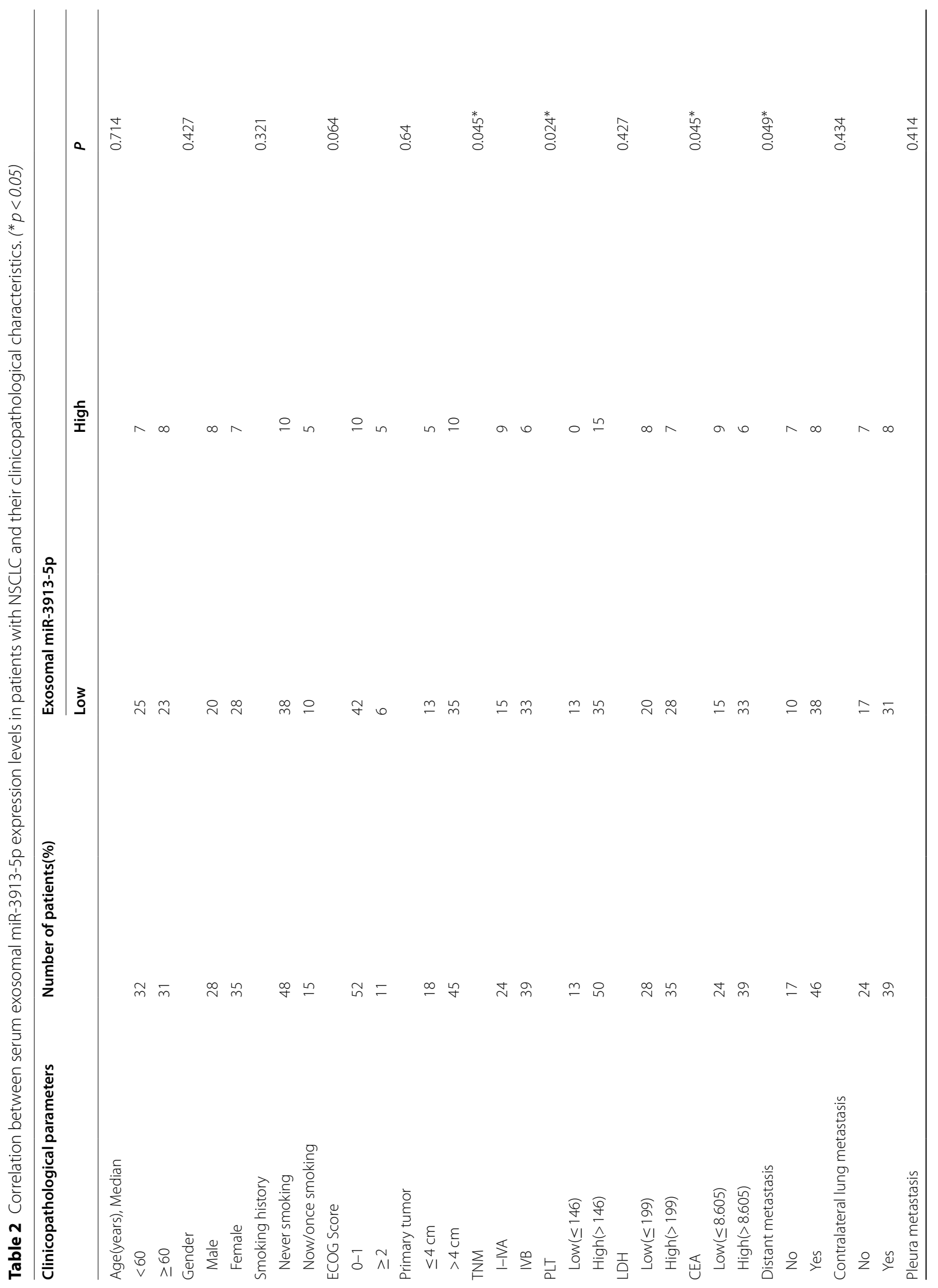




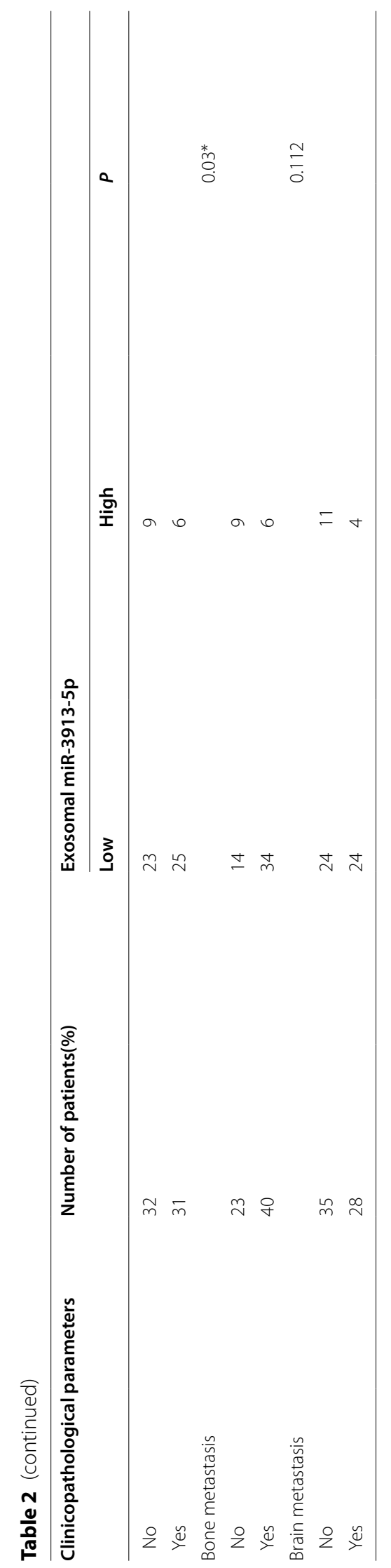




\section{A \\ EGFR 21 L858R \\ Exo-miR-184}

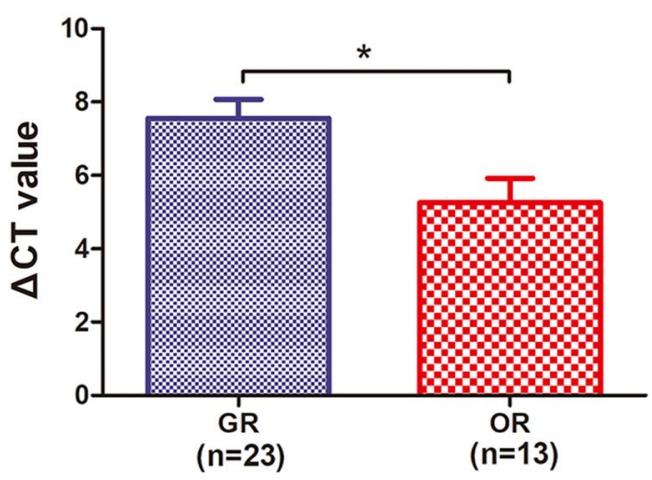

C

$\mathrm{T} 790 \mathrm{M}^{+}$

Exo-miR-184

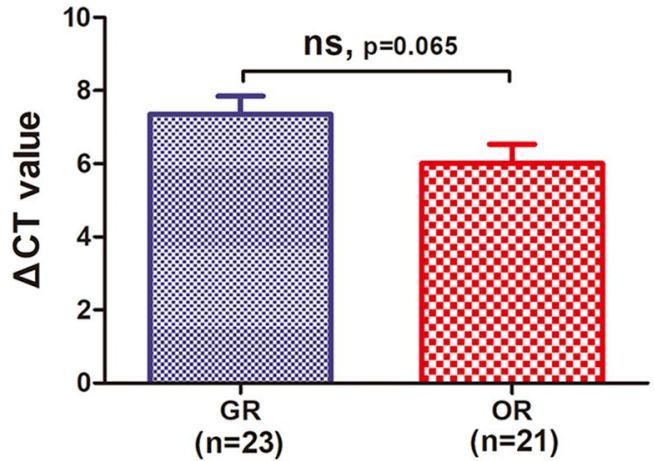

B
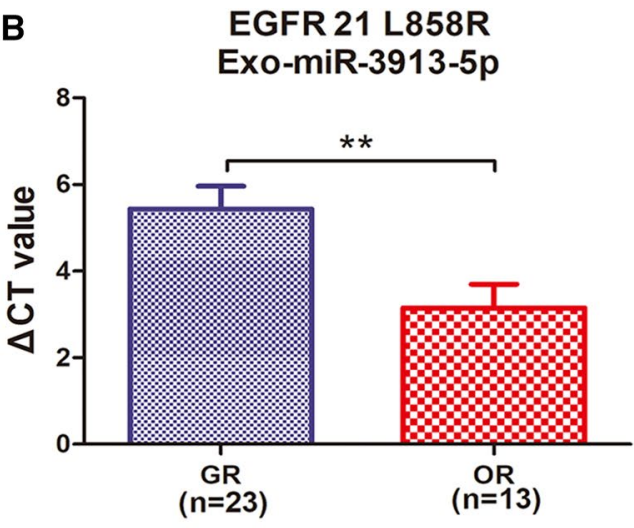

$\mathrm{T} 790 \mathrm{M}^{+}$

Exo-miR-3913-5p

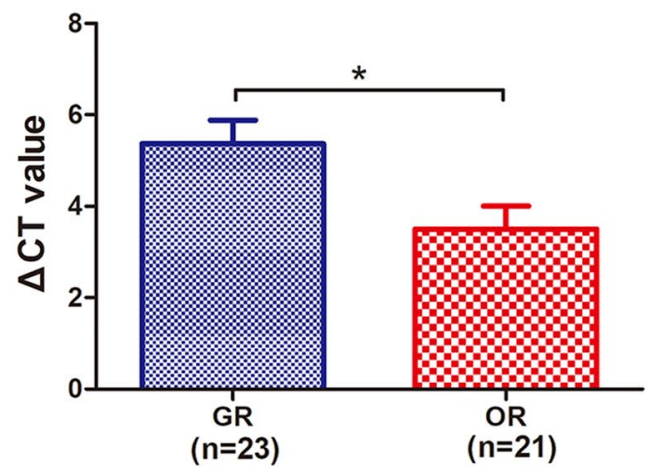

Fig. 4 Osimertinib resistance induced by exosomal miRNAs targeting EGFR 21 mutations and T790M + patients. A q-PCR analysis of serum exosomal miR-184 and B miR-3913-5p expression levels in patients with drug resistance and sensitivity, in all patients with EGFR 19 exon deletion. $\mathbf{C}$ q-PCR analysis of serum exosomal miR-184 and $\mathbf{D}$ miR-3913-5p expression levels in patients with resistance and sensitivity in all patients with EGFR 19 exon L858R mutation

participated in osimertinib resistance by having an impact on bypass activation.

As mentioned previously, exosomes can contain a large number of proteins, nucleic acids, and lipids, which transmit information between cells [28]. Tumor-derived exosomes can be detected in the blood and body fluids of patients. It has been demonstrated that exosomes can affect the therapeutic response and induce drug resistance in tumor cells [29]. Recent research has suggested that drug-resistant cells transmit drug resistance information to drug-sensitive cells through extracellular vesicles [30]. The miRNAs, which are short non-coding RNA, have been thoroughly studied in oncology [31]. When miRNAs are loaded into exosomes, they can be protected from degradation by RNases [32]. An interesting finding was the discovery that exo-miRNAs could assist in the diagnosis of NSCLC. We compared the levels of exosome miR-184, miR-3913-5p, miR-3614-5p, and miR-4746-5p in the serum of NSCLC patients and healthy individuals, and found significant differences between the two. The area under the curve (AUC) of miR-184 was 0.803 (95\% confidence interval: $0.701-0.905)$, greater than 0.75 , indicating that miR-184 of serum exosomes of lung cancer patients could be used as a biomarker for the diagnosis of NSCLC (Additional file 1: Figure S5).

In recent years, many papers have been published on the relationship between exosome derived miRNAs and drug resistance in the treatment of NSCLC [33, 34]. However, few studies have discussed the relationship between the resistance to the third-generation EGFR-TKI osimertinib and exosomes. In our study, we observed exosomes extracted from the cultured H1975 and H1975-OR cell supernatant and the serum of three patients. Changes in the expression levels of exo-miRNAs after osimertinib resistance were captured. We identified, for the first time, that the increased levels of miR-184 
and miR-3913-5p can predict resistance to osimertinib in NSCLC patients treated with this drug. In the clinicopathological features, LDH has been proven to be closely related to clinical prognosis in a variety of malignant tumors [35]. NSCLC patients with higher LDH levels have a worse prognosis and shorter survival than those with lower levels [36]. Although the exosome miR-184 found in our study was related to LDH level $(p=0.018)$ because of the time limit of this experiment, we could not continue to follow up to obtain survival data; therefore, whether exosome miR-184 could be a prognostic indicator remains unclear. CEA is the most common biomarker of lung adenocarcinoma [37]. It has been confirmed that an increase in CEA levels during TKI treatment for EGFR mutation patients may be a more sensitive predictor of an explosive progression in lung adenocarcinoma [38]. PLT is often associated with the platelet-to-lymphocyte ratio (PLR) [39]. Studies have suggested that the preoperative PLT-PLR score could be of significance in predicting the prognosis of patients with surgically resected NSCLC [40]. In this study, we found that exo-miR-3913-5p was related to TNM stage $(p=0.045)$, PLT $(p=0.024)$, CEA $(p=0.045)$, distant metastasis $(p=0.049)$, and bone metastasis $(\mathrm{p}=0.03)$. Moreover, the AUC in the receiver operating characteristic curve was greater than 0.75 , which further suggested that the exosome miR-3913-5p expression level was associated with advanced progression of lung adenocarcinoma in patients with EGFR mutations during TKI use.

Venn diagram analysis demonstrated that miR-184 expression level alteration was overlapped in Treat1 (H1975 lines cells) and Treat4 (A patient with EGFR exon 19 del). MiR-3913-5p expression level alteration was found to be overlapped in Treat 3 and Treat4, and both patients had EGFR exon 19 deletions. However, we found in these subgroup analyses that the expression levels of both miRNAs were significantly altered in patients with EGFR exon 21 L858R point mutations. Previous studies have found that exon 19 deletion mutations (55.0\%) have a higher rate of T790M resistance mutations than exon 21 L858R point mutations (37.3\%) [41]. Recent studies have shown that the hazard ratio of survival benefit for Asian and L858R mutant populations is close to 1.00 in all people receiving osimertinib treatment [6]. Our study found that the expression levels of these two exo-miRNAs changed significantly in the L858R mutant population; however, this phenomenon was not observed in the population with exon 19 deletion. Thus, these two exosome-derived miRNAs might be more useful to predict resistance to osimertinib in patients with EGFR exon 21 L858R point mutations than in other populations. Previous studies have reported that nearly half of the patients lost the T790M mutation at the time of progression to osimertinib [42], and this loss may be related to the early resistance to osimertinib. The loss of T790M mutation is not conducive to prognosis [43]. Plasma T790M levels may predict acquired resistance [44]. However, considering that osimertinib is selective for EGFR sensitivity and T790M mutation, scholars believe that the emergence of a T790M mutation under osimertinib treatment is not a drug-resistance mechanism [19]. However, in this study, we found that exosomal miR-3913-5p expression levels were significantly altered in T790M-positive patients, indicating that this exo-miRNA may be involved in the drug-resistance mechanism in such patients. Exosomal miR-184 and miR-3913-5p are likely to be important molecules for the transmission of osimertinib resistance.

Our study had some limitations. The number of patients validated in this study was 64 , and the number of collected specimens was not ideal. Only patient serum samples were used to extract exosomes, and no further humoral exosomes were used for verification. The clinical characteristics of the patients were collected, but no survival analysis was performed owing to the limitation of followup time. The predicted target genes and pathways will be verified in our subsequent experiments.

Our experiment demonstrated that exosome-derived miRNAs may be involved in the mechanisms of resistance to the third-generation EGFR-TKI osimertinib, especially affecting bypass pathway activation. Therefore, an increase in the expression levels of exosome-derived miR184 and miR-3913-5p in the peripheral blood of NSCLC patients receiving osimertinib treatment may indicate the development of drug resistance. We hope that this study helps to expand the application of liquid biopsy technology in the field of clinical drug resistance in lung cancer. This may not only favorably guide clinical treatment but also provide direction for the development of a new generation of targeted drugs.

\section{Abbreviations}

NSCLC: Non-small cell lung cancer; TKI:Tyrosine kinase inhibitor; EGFR: Epidermal growth factor receptor; BRAF: Serine/threonine protein kinase b-Raf; T790M: Thr790Met; DMSO: Dimethyl sulfoxide; FBS: Fetal bovine serum; PLT: Platelet count; LDH: Lactate dehydrogenase; CEA: Carcinoembryonic antigen.

\section{Supplementary Information}

The online version contains supplementary material available at https://doi. org/10.1186/s12935-021-02075-8.

Additional file 1: Figure S1: (A) and (B) particle size analysis of exosomes in the cell supernatant and plasma of patients, respectively. Figure S2: Pathway enrichment analysis of differentially expressed miRNA target genes. Figure S3. Differentially expressed exosome-derived miRNAs before and after osimertinib resistance. Figure S4. Exosome-derived miRNAs related to osimertinib resistance in patients with T790M mutation. Figure S5. Diagnostic value of exosomal miRNAs for NSCLC. Table S1. Exosomal 
miRNAs-induced osimertinib resistance by the activation of bypass pathways.

\section{Acknowledgements}

The authors would like to extend their sincere gratitude to the reviewers.

\section{Authors' contributions}

$\mathrm{XL}$ and $\mathrm{TL}$ elaborated the initial experimental design. CC completed the design of the overall experimental scheme. $X \mathrm{~L}$ wrote the first draft of the article. ZW, JL, and WS completed the drawing of pictures and tables. MC participated in the extraction of exosomes. XL, CC, and SZ completed exosome identification and extended sample validation experiments. KS and YL conducted the data analysis. PZ conceived the project. TL and YS supervised the research. All authors edited the final manuscript together. All authors read and approved the final manuscript.

\section{Funding}

This work was supported by Grants from the National Natural Science Foundation of China (Grant Numbers 81772500, 81770082, and 81572273) and the Key Research and Development Program of Jiangsu Province (Grant Number BK2019719).

\section{Availability of data and materials}

The datasets used and/or analyzed during this study are available from the corresponding author upon reasonable request.

\section{Declarations}

\section{Ethics approval and consent to participate}

The research protocol was reviewed and approved by the Ethical Committee and Institutional Review Board of the Jinling Hospital affiliated to Nanjing University School of Medicine, and written informed consent was obtained from each patient included in the study (Ethical code:2017NZGKJ-030).

\section{Consent for publication}

Not applicable.

\section{Competing interests}

The authors declare that they have no competing interests.

\section{Author details}

${ }^{1}$ Department of Respiratory and Critical Care Medicine, Jinling Hospital, Nanjing University School of Medicine, 305 East Zhongshan Road, Nanjing 210002, Jiangsu, China. ${ }^{2}$ Department of Respiratory and Critical Care Medicine, Nanjing Drum Tower Hospital, Nanjing University School of Medicine, Nanjing, China. ${ }^{3}$ Department of Respiratory and Critical Care Medicine, Jinling Hospital, The first School of Clinical Medicine, Southern Medical University (Guangzhou), Nanjing, China. ${ }^{4}$ Department of Respiratory and Critical Care Medicine, Jinling Hospital, Medical School of Southeast University, Nanjing, China. ${ }^{5}$ Nanjing University Institute of Respiratory Medicine, Nanjing, China.

\section{Received: 24 February 2021 Accepted: 5 July 2021}

\section{Published online: 14 August 2021}

\section{References}

1. Takano T. Epidermal growth factor receptor gene mutations and increased copy numbers predict gefitinib sensitivity in patients with recurrent non-small-cell lung cancer. J Clin Oncol. 2005;23(28):6829-37.

2. Shi Y, Li J, Zhang S, Wang M, Yang S, Ning L, et al. Molecular epidemiology of EGFR mutations in asian patients with advanced non-small-cell lung cancer of adenocarcinoma histology-Mainland China subset analysis of the PIONEER study. PLoS ONE. 2015;10(11):e0143515.

3. Kumarakulasinghe NB, van Zanwijk N, Soo RA. Molecular targeted therapy in the treatment of advanced stage non-small cell lung cancer (NSCLC). Respirology (Carlton, Vic). 2015;20(3):370-8.
4. Maemondo M, Inoue A, Kobayashi K, Sugawara S, Oizumi S, Isobe H, et al. Gefitinib or chemotherapy for non-small-cell lung cancer with mutated EGFR. N Engl J Med. 2010;362(25):2380-8.

5. Cross DA, Ashton SE, Ghiorghiu S, Eberlein C, Nebhan CA, Spitzler PJ, et al. AZD9291, an irreversible EGFR TKI, overcomes T790M-mediated resistance to EGFR inhibitors in lung cancer. Cancer Discov. 2014;4(9):1046-61.

6. Ramalingam SS, Vansteenkiste J, Planchard D, Cho BC, Gray JE, Ohe Y, et al. Overall survival with osimertinib in untreated, EGFR-mutated advanced NSCLC. N Engl J Med. 2020;382(1):41-50.

7. Schorey JS, Bhatnagar S. Exosome function: from tumor immunology to pathogen biology. Traffic (Copenhagen, Denmark). 2008;9(6):871-81.

8. van der Pol E, Boing AN, Harrison P, Sturk A, Nieuwland R. Classification, functions, and clinical relevance of extracellular vesicles. Pharmacol Rev. 2012;64(3):676-705.

9. Valadi H, Ekstrom K, Bossios A, Sjostrand M, Lee JJ, Lotvall JO. Exosomemediated transfer of mRNAs and microRNAs is a novel mechanism of genetic exchange between cells. Nat Cell Biol. 2007;9(6):654-9.

10. Falcone G, Felsani A, D'Agnano I. Signaling by exosomal microRNAs in cancer. J Exp Clin Cancer Res. 2015;34:32.

11. Yu DD, Wu Y, Zhang XH, Lv MM, Chen WX, Chen X, et al. Exosomes from adriamycin-resistant breast cancer cells transmit drug resistance partly by delivering miR-222. Tumour Biol. 2016;37(3):3227-35.

12. Alharbi M, Zuniga F, Elfeky O, Guanzon D, Lai A, Rice GE, et al. The potential role of miRNAs and exosomes in chemotherapy in ovarian cancer. Endocr Relat Cancer. 2018;25(12):R663-85.

13. Li XY, Huang LT, Wu JQ, He MF, Zhu SH, Zhan P, et al. Zebrafish xenograft model of human lung cancer for evaluating osimertinib resistance. Biomed Res Int. 2019;2019:3129748.

14. Gao W, Lu C, Chen L, Keohavong P. Overexpression of CRM1: a characteristic feature in a transformed phenotype of lung carcinogenesis and a molecular target for lung cancer adjuvant therapy. J Thorac Oncol. 2015;10(5):815-25.

15. Théry C, Amigorena S, Raposo G, Clayton A. Isolation and characterization of exosomes from cell culture supernatants and biological fluids. Curr Protoc Cell Biol. 2006; Chapter 3: Unit 3.22.

16. Conde E, Angulo B, Tang M, Morente M, Sanchez-Cespedes M. Molecular context of the EGFR mutations: evidence for the activation of mTOR/S6K signaling. Clin Cancer Res. 2006;12(3 Pt 1):710-7.

17. Minari R, Bordi P, Tiseo M. Third-generation epidermal growth factor receptor-tyrosine kinase inhibitors in T790M-positive non-small cell lung cancer: review on emerged mechanisms of resistance. Transl Lung Cancer Res. 2016:5(6):695-708.

18. Neel DS, Bivona TG. Resistance is futile: overcoming resistance to targeted therapies in lung adenocarcinoma. NPJ Precis Oncol. 2017;1(1):3.

19. Leonetti A, Sharma S, Minari R, Perego P, Giovannetti E, Tiseo M. Resistance mechanisms to osimertinib in EGFR -mutated non-small cell lung cancer. Br J Cancer. 2019;121:1-13.

20. Bollinger M, Agnew A, Mascara G. Osimertinib: a third-generation tyrosine kinase inhibitor for treatment of epidermal growth factor receptormutated non-small cell lung cancer with the acquired Thr790Met mutation. J Oncol Pharm Pract. 2017:24:107815521771240.

21. MokT, Wu Y-L, Ahn M-J, Garassino M, Kim HR, Ramalingam S, et al. Osimertinib or platinum-pemetrexed in EGFR T790M-positive lung cancer. N Engl J Med. 2016;376:629-40

22. Yang Z, Yang N, Ou Q, Xiang Y, Jiang T, Wu X, et al. Investigating novel resistance mechanisms to third-generation EGFR tyrosine kinase inhibitor osimertinib in non-small cell lung cancer patients. Clin Cancer Res. 2018. https://doi.org/10.1158/1078-0432.CCR-17-2310.

23. Yu HA, Arcila ME, Rekhtman N, Sima CS, Zakowski MF, Pao W, et al. Analysis of tumor specimens at the time of acquired resistance to EGFR-TKI therapy in 155 patients with EGFR-mutant lung cancers. Clin Cancer Res. 2013:19(8):2240-7.

24. Planchard D, Loriot Y, Andre F, Gobert A, Auger N, Lacroix L, et al. EGFR independent mechanisms of acquired resistance to AZD9291 in EGFR T790M-positive NSCLC patients. Ann Oncol. 2015;26:2073-8.

25. Ortiz-Cuaran S, Scheffler M, Plenker D, Dahmen I, Scheel A, FernándezCuesta L, et al. Heterogeneous mechanisms of primary and acquired resistance to third-generation EGFR inhibitors. Clin Cancer Res. 2016:22:4837-47. 
26. Kim T, Song A, Kim D-W, Kim S, Ahn Y-O, Keam B, et al. Mechanisms of acquired resistance to AZD9291, a mutation-selective Irreversible EGFR Inhibitor. J Thoracic Oncol. 2015;10:1736-44.

27. Blakely CM, Watkins TBK, Wu W, Gini B, Chabon JJ, McCoach CE, et al. Evolution and clinical impact of co-occurring genetic alterations in advanced-stage EGFR-mutant lung cancers. Nat Genet. 2017;49(12):1693-704

28. Regev-Rudzki N, Wilson DW, Carvalho TG, Sisquella X, Coleman BM, Rug $M$, et al. Cell-cell communication between malaria-infected red blood cells via exosome-like vesicles. Cell. 2013;153(5):1120-33.

29. Liu X, Jiang T, Li X, Zhao C, Li J, Zhou F, et al. Exosomes transmit T790M mutation-induced resistance in EGFR-mutant NSCLC by activating PI3K AKT signalling pathway. J Cell Mol Med. 2020;24(2):1529-40.

30. Sousa D, Lima RT, Vasconcelos MH. Intercellular transfer of cancer drug resistance traits by extracellular vesicles. Trends Mol Med. 2015;21(10):595-608.

31. To KK. MicroRNA: a prognostic biomarker and a possible druggable target for circumventing multidrug resistance in cancer chemotherapy. J Biomed Sci. 2013;20:99.

32. Koga Y, Yasunaga M, Moriya Y, Akasu T, Fujita S, Yamamoto S, et al. Exosome can prevent RNase from degrading microRNA in feces. J Gastrointest Oncol. 2011;2(4):215-22.

33. Qin X, Yu S, Zhou L, Shi M, Hu Y, Xu X, et al. Cisplatin-resistant lung cancer cell-derived exosomes increase cisplatin resistance of recipient cells in exosomal miR-100-5p-dependent manner. Int J Nanomed. 2017;12:3721-33

34. Zhang Y, Li M, Hu C. Exosomal transfer of miR-214 mediates gefitinib resistance in non-small cell lung cancer. Biochem Biophys Res Commun. 2018;507(1-4):457-64

35. Valvona CJ, Fillmore HL, Nunn PB, Pilkington GJ. The Regulation and function of lactate dehydrogenase a: therapeutic potential in brain Tumor. Brain Pathol (Zurich, Switzerland). 2016;26(1):3-17.

36. Zhang X, Guo M, Fan J, Lv Z, Huang Q, Han J, et al. Prognostic significance of serum LDH in small cell lung cancer: a systematic review with metaanalysis. Cancer Biomark. 2016;16(3):415-23.
37. He G, Jiang Z, Xue S, Sun X, Wang W. Expression of LDH and CEA in serum in the process of targeted therapy of lung adenocarcinoma and the association between them and prognosis. Oncol Lett. 2019;17(5):4550-6.

38. Gao Y, Song P, Li H, Jia H, Zhang B. Elevated serum CEA levels are associated with the explosive progression of lung adenocarcinoma harboring EGFR mutations. BMC Cancer. 2017;17(1):484.

39. Zhang H, Gao L, Zhang B, Zhang L, Wang C. Prognostic value of platelet to lymphocyte ratio in non-small cell lung cancer: a systematic review and meta-analysis. Sci Rep. 2016;6:22618.

40. Kim SH, Lee HW, Go SI, Lee SI, Lee GW. Clinical significance of the preoperative platelet count and platelet-to-lymphocyte ratio (PLT-PLR) in patients with surgically resected non-small cell lung cancer. Oncotarget. 2016;7(24):36198-206.

41. Huang YH, Hsu KH, Tseng JS, Chen KC, Hsu CH, Su KY, et al. The Association of Acquired T790M Mutation with Clinical Characteristics after Resistance to First-Line Epidermal Growth Factor Receptor Tyrosine Kinase Inhibitor in Lung Adenocarcinoma. Cancer Res Treat. 2018;50(4):1294-303.

42. Papadimitrakopoulou V, Wu YL, Han JY, Ahn MJ, Ramalingam S, John T, et al. LBA51Analysis of resistance mechanisms to osimertinib in patients with EGFR T790M advanced NSCLC from the AURA3 study. Ann Oncol. 2018. https://doi.org/10.1093/annonc/mdy424.064.

43. Lin CC, Shih JY, Yu CJ, Ho CC, Liao WY, Lee JH, et al. Outcomes in patients with non-small-cell lung cancer and acquired Thr790Met mutation treated with osimertinib: a genomic study. Lancet Respir Med. 2018;6(2):107-16

44. Oxnard GR, Hu Y, Mileham KF, Husain H, Costa DB, Tracy P, et al. Assessment of resistance mechanisms and clinical implications in patients With EGFR T790M-positive lung cancer and acquired resistance to osimertinib. JAMA Oncol. 2018;4(11):1527-34.

\section{Publisher's Note}

Springer Nature remains neutral with regard to jurisdictional claims in published maps and institutional affiliations.
Ready to submit your research? Choose BMC and benefit from:

- fast, convenient online submission

- thorough peer review by experienced researchers in your field

- rapid publication on acceptance

- support for research data, including large and complex data types

- gold Open Access which fosters wider collaboration and increased citations

- maximum visibility for your research: over $100 \mathrm{M}$ website views per year

At BMC, research is always in progress.

Learn more biomedcentral.com/submissions 